\title{
ON SOME PROJECTION METHODS FOR THE SOLUTION OF NONLINEAR EQUATIONS WITH NONDIFFERENTIABLE OPERATORS
}

\author{
IOANNIS K. ARGYROS
}

\begin{abstract}
We consider a nonlinear equation with a nondifferentiable operator in a Banach space. We approximate a solution of the nonlinear equation using an iteration, whose iterates can be obtained by solving a certain operator equation in a finite dimensional space.
\end{abstract}

\section{Introduction}

We study the problem of approximating a fixed point $x^{*}$ of the equation

$$
x=F(x)
$$

in a Banach space $E$, where $F$ is a nondifferentiable continuous operator defined on some convex subset $D \subset E$ with values in $E$. Let $F_{1}$ be another continuous operator defined on $E$ with values in $E$, and let $P$ be a linear projection operator $\left(P=P^{2}\right)$ which projects $E$ on its subspace $E_{P}$ and set $Q=I-P$. We will assume that the operator $P F_{1}$ is Fréchet differentiable on $D \subset E$.

We will approximate a fixed point $x^{*}$ of equation (1), using the approximations

$$
x_{n+1}=F\left(x_{n}\right)+P F_{1}^{\prime}\left(x_{n}\right)\left(x_{n+1}-x_{n}\right), \quad n=0,1,2, \ldots
$$

Received Feburary 8, 1990; revised July 30, 1992.

(1980) A.M.S. classification codes: 49D15, 47H17, 65J15, 65B05.

Key words and phrases: Banach space, operator equation, projection operator. 
for some $x_{0} \in D$, where $P F_{1}^{\prime}\left(x_{n}\right)$ is the Fréchet derivative of $P F_{1}(x)$ at $x_{n}$. The iteration (2) has been considered by many authors when $P=I$, the identity operator [4], [7], [8], [9], or when $P=\mathbb{I}$ and $F=F_{1}$ [1], [2], [3], [5], [6].

In this paper we assume that for $x_{0} \in D$ the inverse $\left(I-P F_{1}^{\prime}\left(x_{0}\right)\right)^{-1}$ exists and for $\lambda \in(0,1]$, the following Hölder-continuity assumptions are satisfied

$$
\begin{aligned}
& \left\|\left(I-P F_{1}^{\prime}\left(x_{0}\right)\right)^{-1}\left[P F_{1}^{\prime}(x)-P F_{1}^{\prime}(y)\right]\right\| \leq c_{1}\|x-y\|^{\lambda}, \\
& \left\|\left(I-P F_{1}^{\prime}\left(x_{0}\right)\right)^{-1}\left[Q F_{1}(x)-Q F_{1}(y)\right]\right\| \leq c_{2}\|x-y\|
\end{aligned}
$$

and

$$
\left\|\left(I-P F_{1}^{\prime}\left(x_{0}\right)\right)^{-1}[G(x)-G(y)]\right\| \leq c_{3}\|x-y\|, G(x)=F(x)-F_{1}(x)
$$

for all $x, y \in D$ and some $c_{1}, c_{2}$ and $c_{3} \geq 0$.

Note that in case of convergence, the iteration (2) converges to a fixed point $x^{*}$ of equation (1). Moreover, the problem of computing the iterates $\left\{x_{n}\right\}, n \geq 0$ is equivalent to solving a system of linear algebraic equations of order at most $N$, where $N=\operatorname{dim}\left(\mathbb{E}_{p}\right)$, if $\mathbb{E}_{P}$ is finite dimensional.

We finally apply our results to solve an integral equation with a nondifferentiable kernel.

\section{Existence-Uniqueness Theorems}

We now provide some sufficient conditions for the convergence of iteration (2) to fixed point of equation (1).

Theorem 1. Assume that the following conditions hold:

(i) for $x_{0} \in \mathbb{D}$ the linear operator $\left(I-P F_{1}^{\prime}\left(x_{0}\right)\right)^{-1}$ exists and

$$
\left\|\left[I-P F_{1}^{\prime}\left(x_{0}\right)\right]^{-1}\left(x_{0}-F\left(x_{0}\right)\right)\right\| \leq \eta
$$

(ii) the operators $P F_{1}^{\prime}(x), Q F_{1}(x)$ and $G(x)$ satisfy the conditions (3)-(5) respectively, for some $\lambda \in(0,1]$; 
(iii) the ball $\bar{U}\left(x_{0}, R_{0}\right)=\left\{x \in E \mid\left\|x-x_{0}\right\| \leq R_{0}\right\} \subset D$ where

$$
\begin{gathered}
\eta \frac{h}{1-h} \leq \mathbb{R}_{0}, \\
h=\frac{b c_{1}}{1+\lambda} \eta^{\lambda}+c, \quad c=b\left(c_{2}+c_{3}\right),
\end{gathered}
$$

and

(iv) the quantities $h, b, c_{1}, c$ and $\mathbb{R}_{0}$ satisfy

$$
h<1, c_{1} R_{0}^{\lambda}<1 \text { and } b \geq \frac{1}{1-c_{1} R_{0}^{\lambda}} .
$$

Then, the iterates generated by (2) are well defined for all $n \geq 0$, remain in $\bar{U}\left(x_{0}, R_{0}\right)$ and converge to a fixed point $x^{*} \in \bar{U}\left(x_{0}, R_{0}\right)$ of equation (1), with

$$
\left\|x_{n}-x^{*}\right\| \leq \eta \frac{h^{n}}{1-h}, \quad n=0,1,2, \ldots
$$

Proof. Using the Banach lemma on invertible operators, (9), (3) and (2) we obtain that the linear operator $I-P F_{1}^{\prime}(x)$ is invertible of $\bar{U}\left(x_{0}, R_{0}\right)$ and

$$
\left\|\left(I-P F_{1}^{\prime}(x)\right)^{-1}\left(I-P F_{1}^{\prime}\left(x_{0}\right)\right)\right\| \leq b .
$$

By (2) we get

$$
\begin{aligned}
e_{n+1}= & \left\|x_{n+1}-x_{n}\right\| \leq\left\|\left[I-P F_{1}^{\prime}\left(x_{n}\right)\right]^{-1}\left(I-P F_{1}^{\prime}\left(x_{0}\right)\right)\right\| \\
& {\left[\left\|\left(I-P F_{1}^{\prime}\left(x_{0}\right)\right)^{-1}\left\{P F_{1}\left(x_{n}\right)-P F_{1}\left(x_{n-1}\right)-P F_{1}^{\prime}\left(x_{n-1}\right)\left(x_{n}-x_{n-1}\right)\right\}\right\|\right.} \\
& +\left\|\left(I-P F_{1}^{\prime}\left(x_{0}\right)\right)^{-1}\left(Q F_{1}\left(x_{n}\right)-Q F_{1}\left(x_{n-1}\right)\right)\right\| \\
& \left.+\left\|\left(I-P F_{1}^{\prime}\left(x_{0}\right)\right)^{-1}\left(G\left(x_{n}\right)-G\left(x_{n-1}\right)\right)\right\|\right] .
\end{aligned}
$$

Using (3)-(5), (11), (12) and the finite difference formula, we get

$$
e_{n+1} \leq \frac{b c_{1}}{1+\lambda} e_{n}^{1+\lambda}+b c_{2} e_{n}+b c_{3} e_{n}=\left[\frac{b c_{1}}{1+\lambda} e_{n}^{\lambda}+c\right] e_{n} .
$$

We will now show using induction on $n$ that

$$
e_{n+1} \leq \eta h^{n+1}, \quad n=0,1,2, \ldots
$$


From (13) for $n=0$, we get using (6) and (8) that $e_{1} \leq \eta h$. Hence, inequality (14) is true for $n=1$.

Let us assume that inequality (14) is true for all $k \leq n$. Then

$$
e_{k+1} \leq\left[\frac{b c_{1}}{1+\lambda} e_{k}^{\lambda}+c\right] e_{k} \leq h \eta h^{k} \leq \eta h^{k+1} .
$$

That is, (14) is true for all $n=0,1,2, \ldots$.

We now assume that $x_{j} \in \bar{U}\left(x_{0}, R_{0}\right)$ for $j=0,1,2, \ldots, n$. Then, we get

$$
\left\|x_{0}-x_{n+1}\right\| \sum_{j=1}^{n+1} e_{j} \leq \eta \sum_{j=1}^{n+1} h^{j}=\eta h \frac{1-h^{k+1}}{1-h} \leq R_{0} .
$$

Hence, $x_{n+1} \in \bar{U}\left(x_{0}, R_{0}\right)$.

Moreover,

$$
\left\|x_{n}-x_{n+k}\right\| \leq \sum_{j=0}^{k} e_{n+j} \leq \eta h^{n} \frac{1-h^{k+1}}{1-h} \leq \eta \frac{h^{n}}{1-h} .
$$

That is, the sequence $\left\{x_{n}\right\}$ is Cauchy in a Banach space and as such it converges to some $x^{*} \in \bar{U}\left(x_{0}, R_{0}\right)$. By taking the limit in (15) we obtain (10). Furthermore, by taking the limit in (2) we obtain that $x^{*}=F\left(x^{*}\right)$.

That completes the proof of the theorem.

We can now prove the following theorem.

Theorem 2. Assume that the following are true:

(i) equation (1) has a fixed point $x^{*} \in \bar{U}\left(x_{0}, R_{1}\right)$;

(ii) the hypotheses ( $i)$ and (ii) of Theorem 1 are true;

(iii) the ball $\bar{U}\left(x_{0}, R_{2}\right) \subset D$ with

$$
R_{2} \geq\left(1+h_{1}\right) R_{1}
$$

where

$$
h_{1}=\frac{b_{1} c_{1}}{1+\lambda} R_{1}^{\lambda}+c<1, \quad c_{1} R_{1}^{\lambda}<1
$$

and

$$
b_{1} \geq \frac{1}{1-c_{1} R_{1}^{\lambda}} .
$$




\section{Applications}

Consider the integral equation of the form

$$
x(t)=\int_{0}^{1} K(t, s, x(s)) d s
$$

in the space $E=C[0,1]$, where $K(t, s, x(s))$ is nondifferentiable on some $S \subset E$. Set

$$
F(x)=\int_{0}^{1} K(t, s, x(s)) d s \text { and } F_{1}(x)=\int_{0}^{1} L(t, s, x(s)) d s,
$$

where $L(t, s, x(s))$ is a differentiable operator on $D$. Then

$$
P F_{1}^{\prime}(x)=\int_{0}^{1} \bar{L}_{x}^{\prime}(t, s, x(s)) d s,
$$

where

$$
\bar{L}(t, s, x(s))=\sum_{i=1}^{m} M_{i}(t) N_{i}(s, x(s))
$$

is a degenerate kernel approximating the function $L(t, s, x)$ on $S$. The operator $\bar{L}$ can be a portion of the Taylor or Fourier series for the operator $L(t, s, x)$ if we consider it as a function of $t$. The iteration (2) can now be written as

$$
\begin{aligned}
x_{n+1}(t)= & \int_{0}^{1} K\left(t, s, x_{n}(s)\right) d s-\int_{0}^{1} \bar{L}_{x}^{\prime}\left(t, s, x_{n}(s)\right) x_{n}(s) d s, \\
& +\int_{0}^{1} \bar{L}_{x}^{\prime}\left(t, s, x_{n}(x)\right) x_{n+1}(s) d s .
\end{aligned}
$$

Set,

$$
f_{n}(t)=\int_{0}^{1} K\left(t, s, x_{n}(s)\right) d s-\int_{0}^{1} \bar{L}_{x}^{\prime}\left(t, s, x_{n}(s)\right) x_{n}(s) d s,
$$

then iteration (17) becomes

$$
x_{n+1}(t)=f_{n}(t)+\sum_{i=1}^{m} M_{i}(t) \int_{0}^{1} N_{i}^{\prime}\left(s, x_{n}(s)\right) x_{n+1}(s) d s,
$$

which can be solved to give a family of equations

$$
c_{j}-\sum_{i=1}^{m} a_{j i} c_{i}=b_{j}, \quad j=1, \ldots, m,
$$


where

$$
\begin{gathered}
c_{j}=\int_{0}^{1} N_{j}^{\prime}\left(s, x_{n}(s)\right) x_{n+1}(s) d s, \quad j=1, \ldots, m, \\
a_{j i}=\int_{0}^{1} N_{j}^{\prime}\left(s, x_{n}(s)\right) M_{i}(s) d s, \quad j=1, \ldots, m, i=1, \ldots, m,
\end{gathered}
$$

and

$$
b_{j}=\int_{0}^{1} N_{j}^{\prime}\left(s, x_{n}(s)\right) f_{n}(s) d s, \quad j=1, \ldots, m .
$$

The above family is a system of linear algebraic equation. If the determinant $D\left(x_{n}\right)$ of this system is not equal to zero, then

$$
c_{i}=\frac{1}{D\left(x_{n}\right)} \sum_{k=1}^{m} D_{k i}\left(x_{n}\right) b_{k}
$$

and

$$
x_{n+1}(t)=f_{n}(t)+\sum_{i=1}^{m} \sum_{k=1}^{m} \frac{D_{k i}\left(x_{n}\right) a_{k i}}{D\left(x_{n}\right)},
$$

where $D_{k i}\left(x_{n}\right)$ is the cofactor of the element in the $i$-th row and $k$-th column of the determinant $D\left(x_{n}\right)$.

Let us suppose now that the operators $\bar{L}_{x}^{\prime}(t, s, x), Q(t, s, x), G(t, s, x)$ and $T(t, s, x)$, where $Q(t, s, x)=L(t, s, x)-\bar{L}(t, s, x), G(t, s, x)=\mathbb{I}(t, s, x)-\mathbb{L}(t, s$, $x)$, and

$$
T(s, x, x)=\frac{1}{D(x)} \sum_{i=1}^{m} \sum_{k=1}^{m} M_{i}(t) D_{k i}(x) N_{k}^{\prime}(s, x),
$$

satisfy the conditions

$$
\begin{aligned}
& \left|\bar{L}_{x}^{\prime}(t, s, x)-\bar{L}^{\prime}(t, s, y)\right| \leq c_{1}(t, s)|x-y|^{\lambda} \\
& |Q(t, s, x)-Q(t, s, y)| \leq c_{2}(t, s)|x-y| \\
& |G(t, s, x)-G(t, s, y)| \leq c_{3}(t, s)|x-y|
\end{aligned}
$$

and

$$
|T(t, s, x)| \leq r_{1}(t, s) \text { on } S \text {. }
$$

Then the constants appearing in Theorem 1 can be estimated as follows:

$$
c_{1} \sup _{t \in[0,1]} \int_{0}^{1} c_{1}(t, s) d s, \quad c_{2} \leq \sup _{t \in[0,1] z} \int_{0}^{1} c_{2}(t, s) d s,
$$


and

$$
c_{3} \leq \sup _{t \in[0,1]} \int_{0}^{1} c_{3}(t, s) d s \text { and } b \leq 1+\sup _{t \in[0,1]} \int_{0}^{1} r_{1}(t, s) d s .
$$

Once this is achieved on a specific example, we then define $c_{1}, c_{2}, c_{3}$, and $b$ to be equal to the quantities appearing at the right hand side of the above inequalities respectively. Then we try various guesses for the starting point $x_{0}$ until we find one that together with the rest of the parameters satisfy the hypotheses (i)-(iv) of Theorem 1. Theorem 1 can then be applied to solve the integral equation.

\section{References}

[1] I. K. Argyros, "On Newton's method and nondiscrete mathematical induction", Bull. Austral. Math. Soc., 38, (1988), 131-140.

[2] M. Balazs and G. Goldner, "On the method of the cord and on a modification of it for the solution of nonlinear operator equations", Stud. Cer. Mat., 20 (1968), 981-990.

[3] F. M. Migovich, "On the convergence of projection-iterative mehtods for solving nonlinear operator equation", Dopov. Akad. Nauk. Ukr. RSR, Ser. A, No. 1, (1970), 20-23.

[4] M. N. Prisyazhnyuk, "The convergence of some approximate methods for solving nonlinear operator equations with nondifferentiable operators", Ukrainskii Mathematicheskii Zhurnal, Vol. 30, No. 2, (1978), 261-265.

[5] W. C. Rheinboldt, "A unified convergence theory for a class of iterative processes", S. I. A. M. J. Numer. Anal. 5 (1968), 42-63.

[6] J. W. Schmidt, "Unter Fehrerschranker für regular-falsi-verfahren", Period. Math. Hung., 9, (1978), 241-247.

[7] T. Yamamoto, "A note on a posteriori error bound of Zabreiko and Nguen for Zincenko's iteration", Numer. Funct. Anal. and Optimiz., 9, (9and 10), (1987), 987-994.

[8] P. P. Zabrejko and D. F. Nguen, "The majorant method in the theory of NewtonKantorovich approximations and the Ptâk error estimates", Numer. Funct. Anal. Op. timiz., 9, (1987), 671-684.

[9] A. I. Zincenko, "Some approximate methods of solving equations with nondifferentiable operators", (Ukrainian). Dopovidi Akad. Nauk. Ukrain., RSR (1963), 156-161.

Department of Mathematical Sciences, New Mexico State University, Las Cruces, NM 88003. U. S. A. 